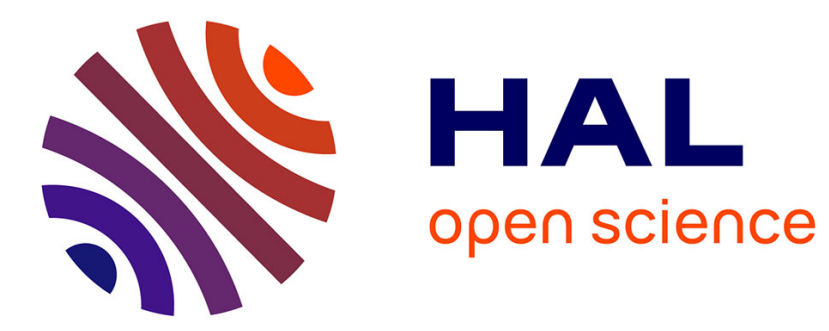

\title{
High density synthesis of topological point defects in graphene on $6 \mathrm{H}-\mathrm{SiC}\left(0001^{-}\right)$
}

Quentin Berrahal, Cyril Chacon, Hakim Amara, Amandine Bellec, Sylvain Latil, Vincent Repain, Sylvie Rousset, Jérôme Lagoute, Yann Girard

\section{- To cite this version:}

Quentin Berrahal, Cyril Chacon, Hakim Amara, Amandine Bellec, Sylvain Latil, et al.. High density synthesis of topological point defects in graphene on $6 \mathrm{H}-\mathrm{SiC}\left(0001^{-}\right)$. Carbon, 2020, 170, pp.174-181. 10.1016/j.carbon.2020.08.008 . hal-02963170

HAL Id: hal-02963170

https: / hal-univ-paris.archives-ouvertes.fr/hal-02963170

Submitted on 6 Nov 2020

HAL is a multi-disciplinary open access archive for the deposit and dissemination of scientific research documents, whether they are published or not. The documents may come from teaching and research institutions in France or abroad, or from public or private research centers.
L'archive ouverte pluridisciplinaire HAL, est destinée au dépôt et à la diffusion de documents scientifiques de niveau recherche, publiés ou non, émanant des établissements d'enseignement et de recherche français ou étrangers, des laboratoires publics ou privés. 


\title{
High density synthesis of topological point defects in graphene on $6 \mathrm{H}-\mathrm{SiC}(000 \overline{1})$
}

\author{
Quentin Berrahal $^{\mathrm{a}}$, Cyril Chacon $^{\mathrm{a}}$, Hakim Amara ${ }^{\mathrm{a}, \mathrm{b}}$, Amandine Bellec $^{\mathrm{a}}$, Sylvain Latil ${ }^{\mathrm{c}}$, Vincent Repain ${ }^{\mathrm{a}}$, Sylvie Rousset ${ }^{\mathrm{a}}$, Jérôme \\ Lagoute $^{\mathrm{a}}$, Yann Girard ${ }^{\mathrm{a}, *}$ \\ ${ }^{a}$ Université de Paris, Laboratoire Matériaux et Phénomènes Quantiques (MPQ), F-75013 Paris, France \\ ${ }^{b}$ Laboratoire d'Etude des Microstructures, ONERA-CNRS, UMR104, Université Paris-Saclay, BP 72, Châtillon, Cedex 92322, France \\ ' Service de Physique de l'Etat Condensé (SPEC), CEA, CNRS UMR 3680, Université Paris Saclay, Orme des Merisiers, CEA Saclay, Gif-sur-Yvette Cedex, 91191, \\ France
}

\begin{abstract}
The control of the physical properties of graphene is a forefront challenge for the development of applications based on this material. Defect engineering is a promising strategy to attain new properties by the insertion of well-defined defects in the carbon lattice. Among the many topological defects of graphene, the one that has the lowest energy per dislocation is a quasi-point-like defect, the so-called Flower Defect (FD). It is generally produced randomly during the graphene synthesis. The deliberate insertion of this defect is promising for the tuning of graphene electronic properties but necessitate to establish a synthesis route allowing to control the formation of flower defects. Here we report a controlled synthesis of graphene with FD using gold as an activator during the graphene synthesis from a $6 \mathrm{H}-\mathrm{SiC}(000 \overline{1})$ silicon carbide wafer. The synthesis consists of a two-step procedure. Creation of a proto-graphene template where one monolayer of gold is evaporated followed by synthesis close to $1200{ }^{\circ} \mathrm{C}$. This leads to a high density of FD, around $10^{12} \mathrm{~cm}^{-2}$. These results are supported by first-principles calculations showing that gold decreases the flower formation energy by a factor of 2. Scanning tunneling spectroscopy measurements reveal unexpected resonances close to the Fermi energy.
\end{abstract}

Keywords: Graphene, Grain Boundaries, Defect engineering, Growth, Silicon carbide, Scanning tunelling microscopy/ spectroscopy, Density functional theory

\section{Introduction}

Control of defect formation (either point-like or extended) in graphene is a challenge as they have long been seen as major drag on the pursuit of good quality graphene. However, they gained interest in the last few years as tuning knobs for the unique properties of graphene [1]. One can, for example, change the position of the Dirac point through atomic substitution (doping) for $\mathrm{n}$ or $\mathrm{p}$ conductivity $[2,3,4,5]$, or create resonant state through single atomic vacancies for monitoring the grafting or functionalization of molecules [6]. Origin of defects in graphene is threefold: they either are native, induced by an external parameter during the synthesis or created afterwards. In polycrystalline graphene, Grain Boundaries (GB) are of the first kind. These 1D defects, mainly composed of pentagons, hexagons, heptagons and vacancies, mark out the separation between two domains of graphene which have different orientation with respect to each other [7, 8]. Having almost all the possible orientations they can eventually form loops encompassing domains of small sizes. They are called Grain Boundary Loops (GBL) and the smallest GBL encompassing 24 atoms, called a one grain boundary loop or a "Flower Defect"(FD), can be seen as a $\pm \pi / 6$ rotation of seven rings inside a graphene grain [9].

\footnotetext{
${ }^{*}$ Corresponding author

Email address: yann.girard@u-paris.fr (Yann Girard)
}

Recently, those FD have attracted great interest towards nanotechnological electronic applications. First, they induce very large modifications of transport properties compared to other point-like defects [10]; second, they create a high spin polarization in zigzag graphene nanoribbon [11]; third, they are able to filter electrons from holes, which have applications for electron energy filtering [12]; fourth, they are good candidates for allelectronic valley based-devices[13]. From theoretical point of view, it is also interesting to note that similar flat geometric defects in honeycomb arrangement have been observed on hexagonal bilayer silica [14] and rubrene on $\mathrm{Au}(111)$ [15]. Nevertheless, their formation on substrates is interesting for applications at wafer scale, but are very rare and rather uncontrolled. So, it is a challenging task to obtain an experimental process which induces selectively a large density of this defect.

Some rare FD appear on various substrates, as a native defect in epitaxial graphene grown on silicon carbide ( $\mathrm{Si}$ rich $\mathrm{SiC}(0001)$ and $\mathrm{C}$ rich $\mathrm{SiC}(000 \overline{1})$ surfaces) [16, 17, 18, 19], as an externally-induced defect during the Chemical Vapor Deposition (CVD) growth of graphene on copper or nickel [4, 20, $21]$, as a result of a post-synthesis annealing in graphene on rhodium [22]. High density of FD, around $10^{11} \mathrm{~cm}^{-2}$, has already been obtained after a few tens of minutes annealing of $\mathrm{SiC}(0001)$ surface around $1250-1350^{\circ} \mathrm{C}$ [23], or assisted by bismuth (Bi) during the graphene synthesis on $\mathrm{SiC}(0001)$ [24]. Nevertheless, FD are always associated with an equivalent den- 
sity of other atomic and nanoscale defects as vacancies, mounds and nanotubes or substitutional atoms [18, 23, 24]. So, none of the existing works provide a procedure to achieve selectively a high density of FD.

We also have to mention that numerous experiments performed on suspended graphene have studied their formation, migration and annihilation under the beam of Transmission Electron Microscopy (TEM) [25, 26]. Associated to theoretical developments [9, 27, 28] and simulations [29], it is now clear that this defect belongs to the family of topological defects conserving both the $s p^{2}$ hybridization character of the C-C bounds and also the number of $\mathrm{C}$ atoms par graphene unit area [30]. The basic units are elementary disclinations (rings of 5 or 7 atoms) which form $5 \mid 7$ pair known as dislocation in order to minimize the elastic energy. The well known Stone-Wales (SW) defect is composed of two dislocations compare to the FD which has six of them. It must be noticed that among all the GB based on those dislocations, FD has the lowest formation energy per dislocation $(1.2 \mathrm{eV})$ [9] because of its nearly full strain relaxation. This explains its stability and its relative frequency of observation. Unfortunately, there is no known atomistic mechanism which could conduct to specific FD formation and simulations are required [29, 31].

In the following, we report Scanning Tunelling Microscopy and Spectroscopy (STM, STS) studies of a gold (Au) assisted two steps growth process that allows to produce selectively a high density of FD in the top Graphene Layer (GL) grown on $6 \mathrm{H}-\mathrm{SiC}(000 \overline{1})$. (Step 1) prepares the surface in order to have connected irregular small graphene islands presenting a high density of borders and stressed areas. We call it proto-graphene. (Step 2) conducts to : (i) extend laterally the previous graphene islands; (ii) form new GL below the first one and (iii) release elastic energy induces by irregular GB. This is completed by : (iv) the fundamental effect of $\mathrm{Au}$ which favors the formation of localized defects. (Step 1) and (step 2) drive together all the parameters which are crucial to obtain a high density of FD. This result is complemented by first-principles calculations which show that Au decreases significantly the FD formation energy. Moreover, we show by fine STS measurements and in depth analysis of FD electronic structure, that those GBL exhibit more localized states than previously reported. STS results are also mentioned concerning resonant states which arise around the Fermi energy $E_{F}$, inside the phonon pseudo-gap [32].

\section{Methods}

\subsection{Sample preparation}

Prior to graphene synthesis (step $1 \& 2)$, the $\operatorname{SiC}(000 \overline{1})$ single-crystals $\left(10 \times 5 \times 0.5 \mathrm{~mm}^{-3}\right.$, C-face epi-ready, Si-Face optically polished) bought from Novasic SA were degassed under ultra-high vacuum (UHV) conditions by direct current heating for 6 hours at $700^{\circ} \mathrm{C}$ before a 15 minute annealing at $850^{\circ} \mathrm{C}$ in front of a $\mathrm{Si}$ evaporator to get rid of surface oxygen. During (step 1 \& 2)'s annealing performed at high temperature, the pressure stays lower than $10^{-8}$ mbar. After annealing, we stopped abruptly the heating power and the temperature decreased within a one minute characteristic time below $300^{\circ} \mathrm{C}$.
Temperatures are measured by a pyrometer in the $1.45-1.80 \mu \mathrm{m}$ spectral bandwidth with a 0.9 emissivity. The sample is maintained laterally by two tantalum stripes on the sample holder and only $1 / 3$ of the sample area is covered by graphene after synthesis. Gold was deposited using an electron beam evaporator EFM 3 (Focus $\mathrm{GmbH}$ ) at a rate of $1.410^{14} \mathrm{Au} / \mathrm{cm}^{2} / \mathrm{min}$, calibrated by Rutherford back scattering.

\subsection{STM/STS measurements}

Except STM pictures reported Fig. 1(a,d) acquired at room temperature (RT), our STM/STS measurements were performed at $4.6 \mathrm{~K}$ using a Scienta Omicron Low Temperature STM running on liquid helium. STS spectra were recorded through lockin detection at frequencies around $700-800 \mathrm{~Hz}$ and with a peakto-peak modulation of $24 \mathrm{mV}$ for the large energy scale spectra and $6 \mathrm{mV}$ for the small energy scale spectra to reduce the broadening of the peaks. Prior to measurements on graphene, tungsten tips were systematically calibrated on $\mathrm{Au}(111)$ until the Shockley surface state is clearly measured in the STS spectra.

\subsection{First-principles calculations}

To investigate the formation energy of the flower defect in presence of $\mathrm{Au}$ atoms, we performed first-principles calculations based on density functional theory. We used the Quantum Espresso [33] code within the projector-augmented wave (PAW) method [34]. The generalized gradient approximation [35] was employed for all calculations. Integrations over the Brillouin zone are based on a $3 \times 3 \times 1$ Monkhorst-Pack threedimensional grid for 162 atom cells. The size of the supercell in the $c$ direction was set to $15 \AA$ to avoid the interaction between neighboring defects. Calculations were performed at zero pressure, i.e., the relaxation of the atoms and the shape of the simulation cell are considered using the conjugate gradient minimization scheme. The initial lattice parameter is equal to $2.45 \AA$ and is allowed to change during the simulations. The atomic positions were relaxed until the magnitude of the forces on all the atoms was smaller than $1.10^{-5} \mathrm{Ha} / \mathrm{Bohr}$. Description of the van der Waals dispersive forces has been accounted for via the D2 dispersion correction of Grimme [35]. No SiC substrate was included in the DFT calculations insofar as our investigation has sought to highlight the intrinsic role played by $\mathrm{Au}$ in flower defect formation. In this context, DFT calculations were performed with various amounts of simulated deposited $\mathrm{Au}$ atoms ranging from 1 to 12 isolated atoms and also a 25-atom Au cluster. Moreover, the adsorption energy, $E_{a d s}$, is defined as :

$$
E_{a d s}=E_{A u / \text { substrate }}-E_{\text {substrate }}-E_{A u},
$$

where $E_{A u / \text { substrate }}$ is the total energy of carbon substrate (graphene or FD) with the Au adatom attached, $E_{\text {substrate }}$ is the total energy of the pristine graphene or the FD layer, and $E_{A u}$ the total energy of an isolated $\mathrm{Au}$ atom. The more negative $E_{a d s}$ is, the more strongly the atom of Au adheres to the carbon substrate. 


\section{Results and Discussion}

\subsection{Gold assisted flower defects synthesis : a two step process studied by scanning tunnelling microscopy}

In order obtain a large amount of FD, we improved usual process where rotationally faulted Few Layer Graphene (FLG) is produced by silicon $(\mathrm{Si})$ sublimation from the substrate annealed between $1100-1300^{\circ} \mathrm{C}[36,37]$. Before our graphene synthesis, the $\mathrm{SiC}(000 \overline{1})$ sample was cleaned in UHV as described in the Methods 2.1 section. Then, after this cleaning preparation, we proceeded in two steps. First step (step 1 in the following), around $1410^{14} \mathrm{Au} / \mathrm{cm}^{2}$, i.e. one monolayer (ML) of gold, is deposited onto the clean SiC substrate at RT (forming three-dimensional islands, not shown) and the sample is then annealed five minutes at an intermediate temperature of $1050^{\circ} \mathrm{C}$ (see Fig. 1(a)), the lowest temperature needed to synthesize graphene [38]. At this point, the graphenization is not complete and one can see trenches between domains which can be seen in the line profile of Fig. 1(b). Those trenches disappear during the step 2 graphene synthesis and are the source of GB $[39,40]$.

Second step (step 2 in the following), around one ML of $\mathrm{Au}$ is deposited at RT once more. This second Au ML follows also a Volmer-Weber (VW) growth mode on graphene at RT [41, 42]. Then, the sample is annealed at three increasing temperatures : $1100^{\circ} \mathrm{C}, 1150^{\circ} \mathrm{C}$ and $1200^{\circ} \mathrm{C}, 5$ minutes each. Fig. 1(c) shows the surface after (step 2) and exhibits three graphene domains separated by rotational GB. Note that the bulk Au melting temperature is $1064^{\circ} \mathrm{C}$ (even lower for nanoparticles of 1-2 nm) and we can suppose that $\mathrm{Au}$ atoms are completely removed from the surface after (step 2) because of evaporation, or diffusion into the bulk [43]. We get FD (circled in red) with a density $n$ around $7 \cdot 10^{11} \mathrm{~cm}^{-2}$. As a FD is composed of 24 atoms, we can also say that $\sim 0.5 \%$ of the atoms are part of FD. The defect-defect first neighbor mean distance is equal to $5.4 \pm 2.3 \mathrm{~nm}$ (the minimum distance is the size of an isolated FD, i.e. around $2 \mathrm{~nm}$ ). This result is consistent with a Poisson distribution where the nearest neighbor mean distance is $1 /(2 \sqrt{n})=6 \mathrm{~nm}$. This shows that FD positions are random. In Fig. 1(c) one can see that larger GBL such as double FD (circled in green) are very scarce with a density around $10^{11} \mathrm{~cm}^{-2}$. Single FD are by far prevailing. This result is consistent with the fact that bigger GBL are less energetically favored than FD [9]. Similar double FD have already been observed and some of them (with a non-conserved number of atoms) have been called conjoined-twin defect [23]. The three graphene domains exhibit Moiré Patterns (MP) with different periodicity (mainly related to the twist angle between the two first graphene layers) and the amounts of FD are nearly equal on each one. On one side, this indicates a homogeneous distribution of FD all over the surface independently of the relative orientation of the underlying GL. On the other side, this shows that FD are not surrounded by the same atomic environment, and consequently, could have different mechanical and electronic properties. Effectively: i) FD belonging to a same grain are more or less in interaction laterally with each other's (and the surrounding GB) and these interactions could induce inhomogeneous stresses, which have a great importance concerning local mechanical properties [44]; ii) FD seem to be randomly distributed, thus their electronic properties should also depend on their positions relatively to the underlying layer. A point which is discussed below in relation with local electronic spectroscopy.

Despite the fact that after this synthesis, either by STM or Auger electron spectroscopy we did not find any trace of $\mathrm{Au}$, we cannot exclude the formation of gold carbides or silicides during the high temperature graphene synthesis. Effectively, AuC complexes have already been observed by TEM [45] and previous calculations [46] have shown that $\mathrm{Au}-\mathrm{C}$ binding energy could be around $0.5 \mathrm{eV}$ higher than the cohesive energy of bulk $\mathrm{Au}(3.81 \mathrm{eV})$. Moreover, we can suppose that during annealing around $1100^{\circ} \mathrm{C}$, as deposited $\mathrm{Au}$ form small liquid droplets before evaporation and constitute temporary sources for $\mathrm{Au}$ atoms/clusters diffusion on the surface, which could also form AuSi complexes.

\subsection{Discussion about the importance of each step}

Starting from the cleaning procedure, all the parameters in this double step process seem to be crucial. For example, we carried out (steps 1 \& 2) without Au deposition and ended up with no FD. This means that Au (especially when deposited during (step 2)) plays a key role in the formation of the FD as catalyst. But proto-graphene is also essential.

\subsubsection{Synthesis without proto-graphene}

If (step 1) is not carried out, i.e. only one Au ML is deposited on the clean $\mathrm{SiC}$ substrate and the sample is then heated gradually until $1200^{\circ} \mathrm{C}$, it exhibits no FD as shown on Fig. 1(d). Effectively, Au melting temperature being lower than $1100^{\circ} \mathrm{C}$, we can assume that most of the Au evaporates from the surface before it can create defects. Thus, this case is similar to the usual FLG synthesis without $\mathrm{Au}$ and our results presented in Fig. 1(d) are comparable to previously obtained results [7, 47]. If we compare Fig.s 1(c) and (d), we remark that without (step 1) the final typical domain size is around five times smaller and the sample exhibits a high density of GB. This is also explained by the proto-graphene which, once created, could reduce the area available for new graphene nucleation sites during the (step 2) synthesis performed at higher temperature $[48,49]$ and so, could increase the final grain size by limiting the proliferation of graphene flake boundaries.

\subsubsection{Proto-graphene as a template for gold anchoring}

The first very important point to notice is that after (step 1) graphene is not flat. As shown in Fig. 1(a,b), (step 1) is not sufficient to create extended graphene domains and the surface is rather similar to graphene obtained on various $\mathrm{SiC}$ reconstructions [50]. The large atomic roughness in Fig. 1(b) indicates the presence of small islands or clusters of undefined species (Au, C, Si or their combination). We think that those areas are one way toward FD synthesis, as they induce locally important strain in the graphene, that increases the elastic energy. 


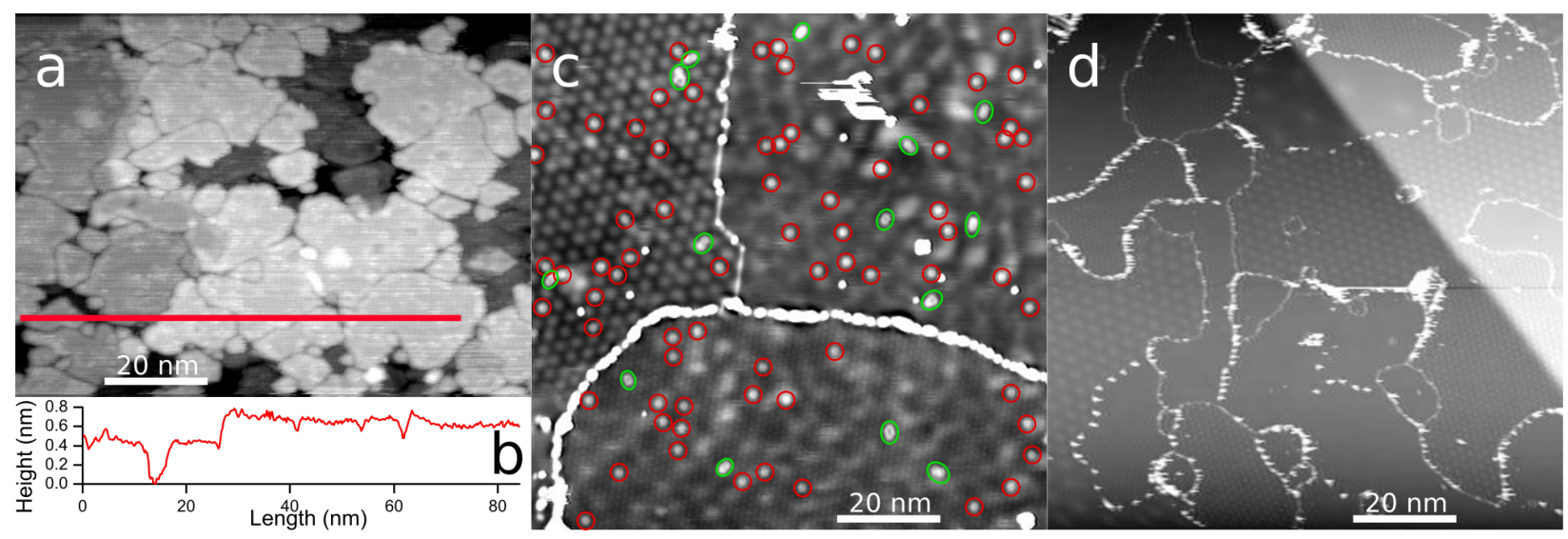

Figure 1: (a) STM image of proto-graphene on $\mathrm{SiC}(000 \overline{1})$ synthesized after (step 1), i.e. : one Au monolayer deposition at room temperature and a 5 minute synthesis at $1050^{\circ} \mathrm{C}\left(100 \times 74 \mathrm{~nm}^{2}\right)$. (b) Topography along the red line showing the trenches between domains. (c) STM image of three domains of few layer graphene obtained after (step 1) followed by (step 2), i.e. : a second Au deposition and three successive 5 minute synthesis at $1100^{\circ} \mathrm{C}, 1150^{\circ} \mathrm{C}$ and $1200^{\circ} \mathrm{C}$. On each of the domains, one have circled in red the flower defects. Bigger grain boundary loops are circled in green $\left(100 \times 100 \mathrm{~nm}^{2}\right) .(\mathrm{d}) \mathrm{STM}$ image of several domains of few layer graphene obtained without (step 1), i.e. : one Au monolayer deposition at room temperature and a 15 minute direct synthesis at $1200^{\circ} \mathrm{C}(100 \times 100$ $\mathrm{nm}^{2}$ ). For all the images $\mathrm{V}=1 \mathrm{~V}, \mathrm{I}=50 \mathrm{pA}$.

During (step 2), this energy will be released locally and consequently may promote a reorganization favorable to the FD. Note also that after (step 1), graphene island borders are connected to the $\mathrm{SiC}$ substrate which induces also a residual stress inside islands [39]. So, we argue that there are two main places where (step 2)'s Au would catalyze defects more easily : the small strained area in the graphene flakes and the graphene borders.

\subsubsection{Gold as catalyst for the flower defects formation}

$\mathrm{Au}$ on graphene at RT follows a VW growth mode but the $\mathrm{Au}$ island density depends strongly on the graphene defect density such as vacancies, graphene borders and GB [42, 51]. All those defects are nucleation areas for island growth [52]. This means that proto-graphene, which exhibits a very high defect density, acts as a perfect template in order to obtain a high density of Au islands during (step 2). We think that it is this association which is the driving force for FD formation. We argue that $\mathrm{Au}$ islands of nanometer size trapped in the proto-graphene defects modify locally the formation/relaxation of graphene and the healing processes during (step 2). One consequence is the generation of small domains isolated from the rest by GBL such as FD or conjoined-twin defects. Containment of small areas within the rest of the environment has already been observed by TEM [12] and a bulge nucleation mechanism has been proposed which has great similarities with our hypothesis. This could be confirmed by molecular dynamic simulations of GB migration $[26,29,53]$ in presence of Au islands.

\subsection{Comparisons with bismuth and platinum}

(Step 2) is carried out at high temperature (around $1150^{\circ} \mathrm{C}$ ) and the stability of Au islands during this step is the key point to increase the FD density. If islands evaporate before the carbon disclinations interact with each other under or at the island edges in order to form GBL, there will be no increase of FD density and no gain relatively to a situation without Au. On the contrary, if Au islands do not evaporate (as observed in the first two annealing at $1100^{\circ} \mathrm{C}$ and $1150^{\circ} \mathrm{C}$, not shown), there could be defects under these islands, but they will obviously be invisible to STM. Since the Au melting temperature is lower than the second graphene synthesis temperature, it is the Au island size, and therefore their lifetime before total evaporation (which is related to the sublimation rate at this temperature) that controls two things. On the one hand, getting FD, and, on the other hand, removing all the residual $\mathrm{Au}$ islands or atoms which could modified the intrinsic flowered-graphene properties. An indirect proof of the importance of the metal melting temperature and the quantity of deposited atoms in (step 2) is to compare our results with those obtained with two other elements which have very different bulk melting temperatures $(\mathrm{BMT})$ : platinum $(\mathrm{Pt})$ and bismuth $(\mathrm{Bi})$ with respective BMT of $1380^{\circ} \mathrm{C}$ and $271^{\circ} \mathrm{C}$, i.e. respectively above and below the (step 2) synthesis temperature. We used Pt instead of $\mathrm{Au}$ in (step 1 \& 2) and, excepted a lot of residual big Pt islands, we didn't obtain any observable FD. Nevertheless, we cannot exclude the possibility that FD are located below those islands. Concerning $\mathrm{Bi}$, we recall below that previous experiments [24] realized on the Si-rich face have also generated a great flower-like density, around $10^{12} \mathrm{~cm}^{-2}$, but associated with a nearly equal amount of undefined point defects. For $\mathrm{Bi}$ on $\mathrm{SiC}(0001)$ [24], (step 1) was not performed and (step 2) was repeated several times with two major differences compared to our experiments : a larger $\mathrm{Bi}$ thickness and a higher temperature, $1350^{\circ} \mathrm{C}$. The consequence was a low amount of potential sites for the nucleation of $\mathrm{Bi}$ islands which could have catalyzed FD formation. Associated with its very low BMT (and so a very high sublimation rate at those temperatures), this explains why (step 2) has to be repeated several times before reaching a significant density of FD. Moreover, it must be pointed out that the graphene growth on the Si-rich surface is intrinsically not a good candidate to obtain selectively a high density of FD. The growth modes of graphene on the Si-rich and C-rich faces are very different. On the former, it is a rolling-carpet mechanism starting at the $\mathrm{SiC}$ step edges, 
while on the latter, it is a nucleation growth mode from the $\mathrm{SiC}$ terraces. It follows that on the Si-rich face, the first successive graphene planes (included the buffer layer) are in epitaxial relationship with the substrate and have very few grain boundaries, whereas on the $\mathrm{C}$-rich face, the graphene planes are rotated relatively to each other, thus forming both MP and having a high density of grain boundaries constituted mainly by disclinations and vacancies. Since flower defects are obtained from the organized clustering of disclinations, it is clear that the more disclinations there are, the greater the density of flower defects will be. However, this is directly related to the density of point defects present during graphene synthesis. It is therefore expected to be more "difficult" to obtain a high density of flower defects on the $\mathrm{Si}$ side compared to the $\mathrm{C}$ side. This is indeed what is observed with bismuth assisted FD formation on the Si-rich face [24] or the necessity to synthetize the graphene at high temperature [23] in order to obtain a high density of defects, among which a certain amount of FD.

\subsection{First-principles calculation of FD formation energy}

Once we have understood that after (step 1) a great amount of potential FD sites (among other extended defects) have been created along graphene flake borders and stressed regions, we theoretically investigate the role of Au during FD formation. So we calculate the FD formation energy, $E_{f}$, by first-principles calculations (see Methods 2.3). We define $E_{f}$ as

$$
E_{f}=E(F D+n \cdot A u)-E(G R+n \cdot A u)
$$

where the first right-hand side term is the total energy of the FD in contact with $n \mathrm{Au}$ atoms, and the second one is the total energy of the corresponding pristine graphene structure also with $n \mathrm{Au}$. To emphasize the catalytic role played by gold to form $\mathrm{FD}, E_{f}$ is calculated for $n \mathrm{Au}$ atoms ( $n=1$ to 12) located in direct contact with the defects. More precisely, our calculations show that the most stable position for one Au atom is in bridge between the pentagonal and heptagonal rings. Therefore, different configurations will be investigated further when the $n \mathrm{Au}$ atoms are close or far from each other on these specific sites. First of all, it can be seen that the formation energy without $\mathrm{Au}$ atom is equal to $7.20 \mathrm{eV}$ which is in agreement with previous results $[9,26]$. Interestingly, our calculations show clearly that $E_{f}$ decreases significantly in presence of Au atoms to reach $3.05 \mathrm{eV}$ for $n=12$. As seen in Fig. 2, the decrease is not continuous. Indeed, it depends on the way Au atoms interact locally with the topological defects present in the graphene. To go further, a flake of $25 \mathrm{Au}$ atoms fully covering the FD is considered. They are forming a closed-packed structure which can be assimilated to the contact zone characterizing the interaction of a (111) facet of a nanoparticle with the FD. By doing so, the decrease in $E_{f}$ is even more pronounced and achieves a value of about $2.5 \mathrm{eV}$. Consequently, those results clearly show that Au promotes formation of FD.

This catalytic role of $\mathrm{Au}$ is achieved through its strong interaction with the topological defects present in graphene. To highlight this mechanism, the adsorption energy $\left(E_{a d s}\right)$ of an $\mathrm{Au}$ atom on a SW defect is calculated and compared with pristine

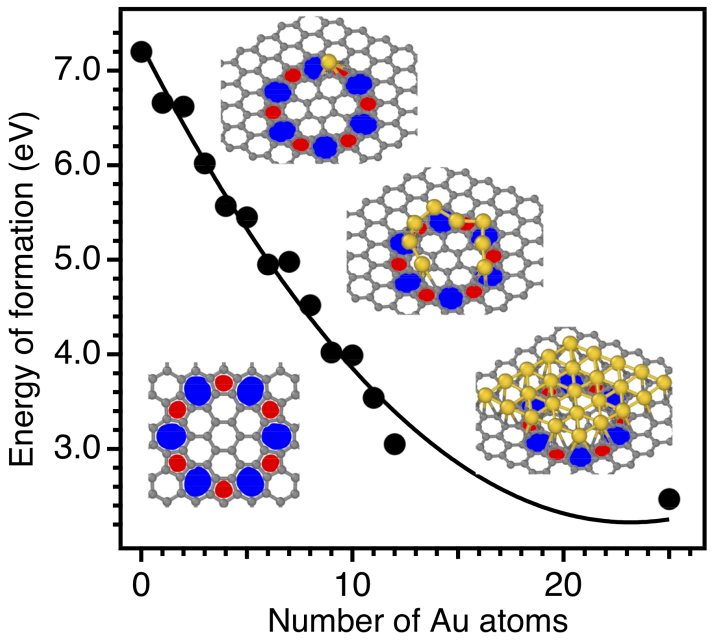

Figure 2: Flower defect formation energy as a function of the number of gold atoms. Insets : relaxed atomic positions of a flower defect without (left) and with 1, 8 and $25 \mathrm{Au}$ atoms (left). Carbon atoms are in black and Au atoms are in yellow. Pentagonal and heptagonal rings are filled in red and blue, respectively.

graphene (see Methods 2.3). The adsorption of $\mathrm{Au}$ atoms on graphene has been studied on several graphene sites, including the highly symmetric sites (top, bridge and hollow). According to our calculations, the most stable positon for the Au adatom is on top of a $\mathrm{C}$ atom for an adhesion energy of about $-0.51 \mathrm{eV}$ in line with data from the literature $[54,55,56]$. In the presence of the SW defect, $E_{a d s}$ is equal to $-2.51 \mathrm{eV}$ revealing that the interaction of $\mathrm{Au}$ atoms with topological defects is very strong which suggests that $\mathrm{Au}$ atoms can play a crucial role for the formation of FD.

It is interesting to note that similar calculations have shown that the energy barrier for the elimination of SW defects (around $5 \mathrm{eV}$ ) [57] can be drastically decreased by adsorbed transition metal atoms $[58,59]$. In the present work, we were also able to highlight this effect. We performed the same kind of study done for FD with a SW defect. In a similar way, we observe a strong reduction of the formation energy in the presence of Au atoms. Indeed, the formation energy of a SW defect is $5.3 \mathrm{eV}$ according to our calculations. In this particular case, the $\mathrm{Au}$ atom occupies the bridge position and tends to strongly decrease the defect formation energy by about $25 \%$ to reach $3.92 \mathrm{eV}$. Starting from 3 atoms, the $E_{f}$ value tends to stabilize around $3 \mathrm{eV}$. Note that once 5/7 pairs or SW defects are formed, by an unknown out of equilibrium atomic process during the graphene formation, they are stable in the temperature range that we use. So, our experimental and theoretical results show that $\mathrm{Au}$, acting as a catalyst, decreases FD formation energy.

Finally, we can say that two main mechanisms act to form a high density of FD. On one hand, Au (and eventually Bi, $\mathrm{Pt}$ and other species) catalyzes FD and flower-like defects because it decreases the SW and loops formation energy. And, on the other hand, (step 1) increases the number of local stressed areas induced by trenches and graphene defects in the protographene, which thus prepares a lot of potential sites for Au anchoring during (step-2) and so favoring the formation of point- 


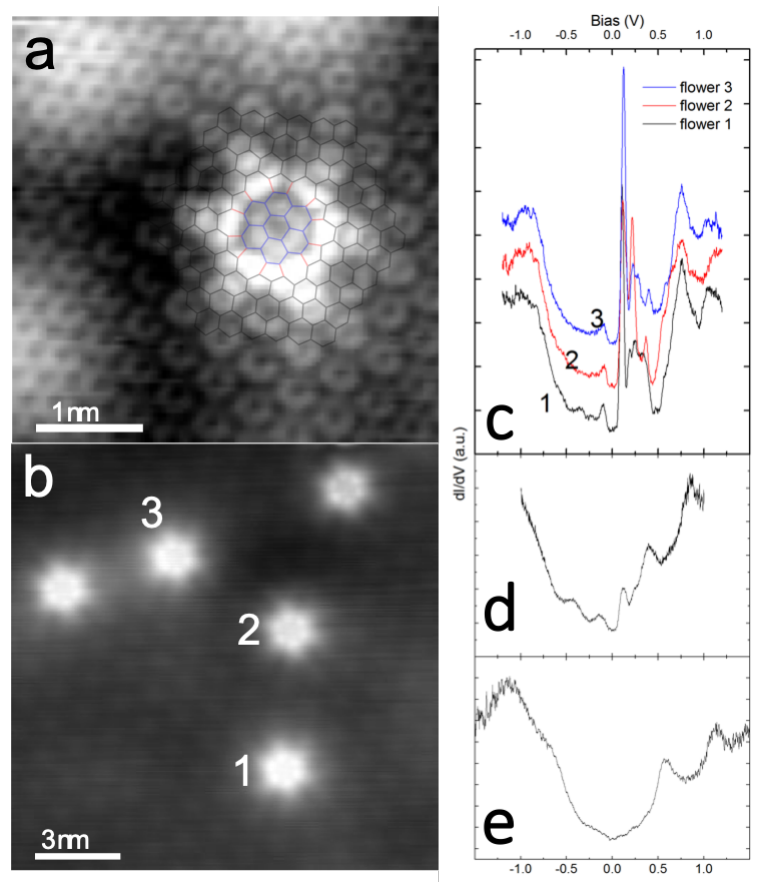

Figure 3: (a) Scanning tunelling microscopy image of a flower defect with the qualitative atomic model superimposed over it $\left(4 \times 4 \mathrm{~nm}^{2}, \mathrm{~V}=1 \mathrm{~V}, \mathrm{I}=100 \mathrm{pA}\right)$.(b) Topographic image of 5 other flower defects $\left(15 \times 15 \mathrm{~nm}^{2}, \mathrm{~V}=220 \mathrm{mV}, \mathrm{I}=\right.$ $1 \mathrm{nA}$ ).(c) $d I / d V$ spectra taken on three of the defects in (b). Offsets have been added for clarity. (d, e) $d I / d V$ spectra taken on FD belonging to two other grains.

like defects.

\subsection{STM/STS characterization of flower defects}

\subsubsection{Topography and spectroscopy at high energy}

The topography of a FD is reported in more details in Fig. 3(a) and its appearance is similar to that previously reported [9, 24] (an atomic model is superimposed to the experimental image). The defect appears as a grey center located on the seven central hexagons, surrounded by a white crown located on the joined $5 \mid 7$ pairs, prolonged by six lobes emerging from the heptagons. Far from the defect (bottom left part), one can see the honeycomb lattice of graphene and closer to it one can see the $(\sqrt{3} \times \sqrt{3}) R 30^{\circ}$ electronic pattern due to electrons scattering by the defect [17]. Note that all apparent heights (and so the grey scale from 0 to $1.1 \AA$ ) are not related to atomic corrugation but mostly to local variations of the electronic densities. Fig. 3(b) shows a STM image of five FD belonging to the same graphene domain exhibiting a unique moiré pattern (MP). Whatever the MP, we never noticed a clear crystallographic relationship between FD location and the apparent moiré periodicity. It means that, on one side, the underlying GL plays no role in the FD location, and, on the other side, that the seven rotated central hexagons are not exactly above the same underlying carbon stacking. Fig. 3(c) shows three differential conductance $(d I / d V)$ spectra taken on three of the FD in Fig. 3(b). There are two broads peaks around $-0.9 \mathrm{eV}$ and $0.8 \mathrm{eV}$ and a series of fine peaks at positive energies between $0.1-0.5 \mathrm{eV}$, just above the pseudo-gap [32] centered at $E_{F}$ (see also Fig. 4(e)). These features in the $\mathrm{eV}$ range are consistent with previous reports $[4,9,22,23,24]$ but our measurements display a better resolved fine structure. More precisely, FD electronic fingerprint is usually observed as a broad resonant peak in the graphene empty states located around 300 to $400 \mathrm{meV}$ above the Dirac Point (DP). This localized resonance is characteristic of isolated SW defects $[60,61]$ and similar to van Hove singularities which emerge in one-dimensional ordered GB [7, 62, 63]. Note that FD which are within the same graphene grain, as in Fig. 3(b), exhibit very similar spectra (this has been systematically observed on several domains) : same peaks at the same energies with slightly different intensities. The number and energy position of these fine FD peaks centered around $0.25 \mathrm{eV}$ in Fig. 3(c) strongly depend on the scanned graphene grain which suggests that these states are sensitive to the surrounding atomic arrangement (see also spectra reported in Fig. 3(d,e) obtained on two different grains, but with a lower resolution), but they are always in the empty states. Correlatively, the mapping of those states is very sensitive to the orientation of underlying GL. Those two observations are a strong indication that FD may interact not only with their lateral atomic environment, but also they couple with the underlying surface. This coupling could explain the multiple peaks structure observed between $0.1-0.5 \mathrm{eV}$ and not predicted by first-principles calculations [9] but presenting great similarities with previous STS measurements performed on flat regular $\mathrm{GB}$ on $\mathrm{SiC}(000 \overline{1})$ [7]. Despite the large energy range we have experimentally explored $\pm 1.5 \mathrm{eV}$, there is no localized state in the filled states.

\subsubsection{Mapping of low energy states}

Zooming the low energy range, i.e. few tens of meV, it is intriguing to see that spectra taken on the different lobes (heptagons) of a FD, see Fig. 4(a), have several clear peaks inside the gap and rather close to $E_{F}$, see Fig. 4(e). We recall that most of the effective electron tunelling in graphene is inelastic electron tunelling mediated by phonons [64] and that a typical STS spectrum on pristine graphene exhibits a gap-like feature (see also Fig. 3(c)) between $-64 \mathrm{mV}$ and $+64 \mathrm{mV}$ (which we confirmed using $d^{2} I / d V^{2}$ measurements). Through an extended study of several defects at different positions on the sample and with different tips we always noticed that diametrically opposed lobes have very similar spectra. Differential conductance maps obtained from grid spectroscopy at different energies are reported in Fig. 4(b-d). That shows that opposing lobes "light up" at the same energy. We also notice that two or four lobes "light up" at the same time but never all six of them. This is surprising in the sense that it breaks the obvious six-fold symmetry that one could expect from the defect. Two possibilities are open. On one hand, the surrounding electronic modulation (induced by defects proximity, anisotropic strain, etc.) could be coupled to those electronic states and thus breaks the sixfold symmetry. On the other hand, FD belonging to the same grain are surrounded by the same MP induced by the twist angle of the graphene bilayer. It follows that the periodic transition from $\mathrm{AB}$ to AA local stackings of the graphene bilayer are locally disturbed by each FD. Thus, because of the FD ran- 

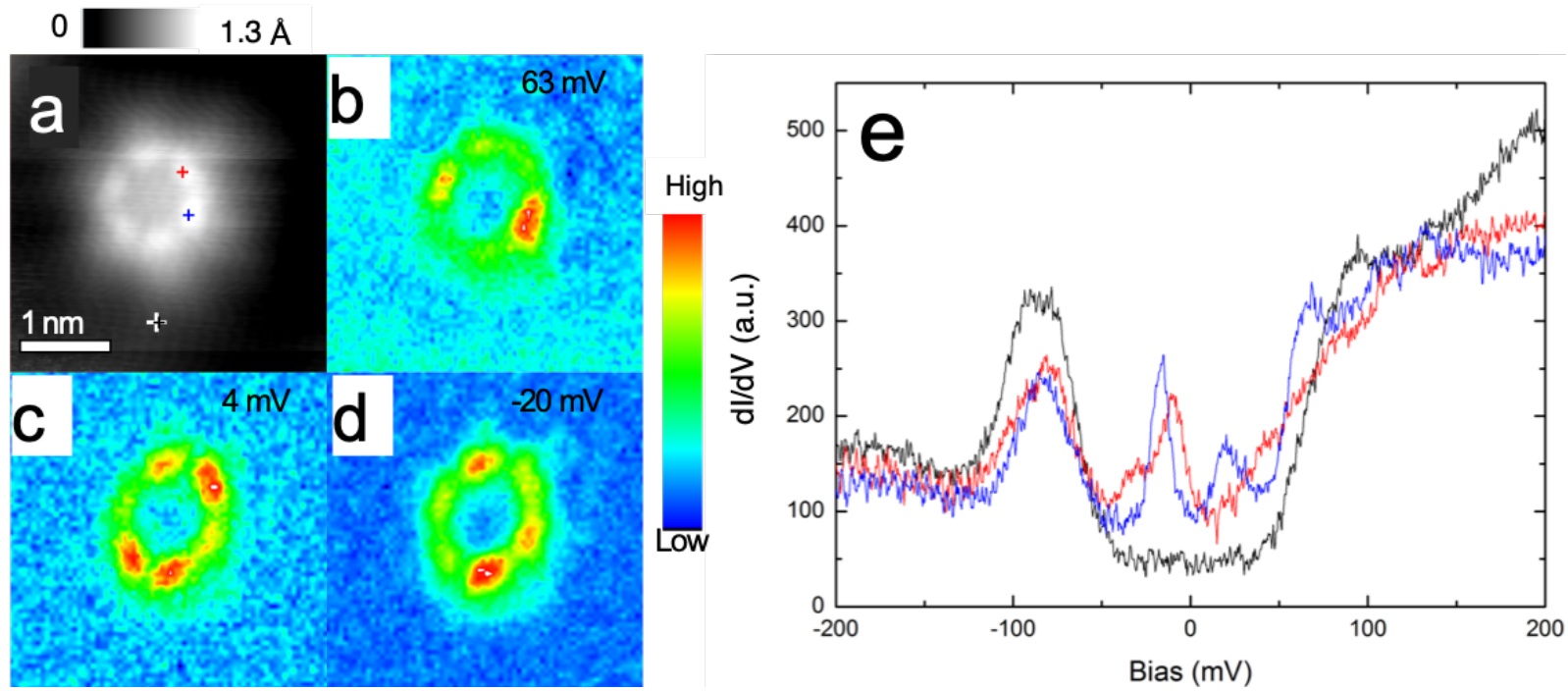

Figure 4: (a) Topographic image of another flower defect $\left(3.5 \times 3.5 \mathrm{~nm}^{2}, \mathrm{~V}=500 \mathrm{mV}, \mathrm{I}=400 \mathrm{pA}\right)$ and differential conductance intensity maps taken from grid spectroscopy at (b) $63 \mathrm{mV}$ (c) $4 \mathrm{mV}$ and (d) $-20 \mathrm{mV}$ on the same defect. (e) $d I / d V$ spectra taken on (a) with a set point at $500 \mathrm{mV}$ and 400 pA. The black curve is taken on the graphene (white ' + ' in (a)) and shows the phonon gap. The red and blue curves are taken on two different lobes of the defect (red and blue ' + 's in (a)) and show both the phonon gap and several peaks inside the gap.

domness location, one should expect variable electronic states from FD to FD. For example, if a flower is in an $A A$ stacking bilayer, its symmetry is not broken, and we should observe a 6-fold symmetry. In case of a Bernal-stacked bilayer we should observe a 3-fold symmetry, as already suggested [9]. Note that a similar symmetry breaking processes induced by a defect in a graphene bilayer has previously been invoked in order to explain the shape of FD [19]. Due to randomness of FD positions in a same grain (and also the same MP), they have not the same underlying stacking (except for the $A A$ or $A B$ stacking which are rather rare), which also breaks their symmetry. This second possibility is the more likely of the two because it is coherent with the spectroscopy done at high energy where all FD residing on the same MP exhibit the same spectrum whatever their proximity with other defects. We can not explain the physical origin of those in-gap states (these states have already been measured in a very different atomic configurations, i.e. along graphene nanoribbons on $\mathrm{Au}(111)$ [65] and along ridges on $\mathrm{SiC}(0001)$ [66])), but the FD special topology coupled to the interaction with the underlying graphene could induce new states due to electronic confinement. Recent calculations on circular quantum dots in twisted bilayer graphene are certainly a hint to understand those states [67].

\section{Conclusion}

In summary, scanning tunelling microscopy and spectroscopy studies of flower defect formation catalyzed by gold on fewlayer graphene on $\mathrm{SiC}(000 \overline{1})$ have been presented. A very high density of flower defects is obtained thanks to a two-steps process. A proto-graphene layer creates a template for anchoring gold clusters which promotes the formation of those quasi-point like topological defects during the second step. First-principles calculations provide clear evidence for a drastic decrease of their formation energy with gold. These flowers exhibit localized electronic states all over the defect at high energy, and interestingly low energy states localized asymmetrically on the heptagons breaking the apparent flower defect 6-fold symmetry. The complex formation of those defects during the graphene synthesis on $\mathrm{SiC}$ could be studied by molecular dynamic simulations. The unknown in-gap states deserve future experiments and theoretical developments.

\section{Acknowledgments}

The authors received no specific funding for this work.

\section{References}

[1] Z. Wu, Z. Ni, Spectroscopic investigation of defects in two-dimensional materials, Nanophot. 6 (6) (2017) 1219-1237 (Mar. 2017). doi:10. 1515/nanoph-2016-0151.

[2] F. Joucken, Y. Tison, J. Lagoute, J. Dumont, D. Cabosart, B. Zheng, V. Repain, C. Chacon, Y. Girard, A. R. Botello-Méndez, S. Rousset, R. Sporken, J.-C. Charlier, L. Henrard, Localized state and charge transfer in nitrogen-doped graphene, Phys. Rev. B 85 (16) (2012) 161408 (Apr. 2012). doi:10.1103/PhysRevB.85.161408.

[3] L. Zhao, R. He, K. T. Rim, T. Schiros, K. S. Kim, H. Zhou, C. Gutiérrez, S. P. Chockalingam, C. J. Arguello, L. Pálová, D. Nordlund, M. S. Hybertsen, D. R. Reichman, T. F. Heinz, P. Kim, A. Pinczuk, G. W. Flynn, A. N. Pasupathy, Visualizing Individual Nitrogen Dopants in Monolayer Graphene, Science 333 (6045) (2011) 999 (Aug. 2011). doi:10.1126/ science. 1208759.

[4] L. Zhao, M. Levendorf, S. Goncher, T. Schiros, L. Pálová, A. ZabetKhosousi, K. T. Rim, C. Gutiérrez, D. Nordlund, C. Jaye, M. Hybertsen, D. Reichman, G. W. Flynn, J. Park, A. N. Pasupathy, Local Atomic and Electronic Structure of Boron Chemical Doping in Monolayer Graphene, Nano Lett. 13 (10) (2013) 4659-4665 (Oct. 2013). doi : 10.1021/nl401781d.

[5] F. Joucken, L. Henrard, J. Lagoute, Electronic properties of chemically doped graphene, Phys. Rev. Materials 3 (11) (2019) 110301 (Nov. 2019). doi:10.1103/PhysRevMaterials.3.110301. 
[6] F. Ducastelle, Electronic structure of vacancy resonant states in graphene: A critical review of the single-vacancy case, Phys. Rev. B 88 (7) (2013) 075413 (Aug. 2013). doi: 10.1103/PhysRevB.88.075413.

[7] Y. Tison, J. Lagoute, V. Repain, C. Chacon, Y. Girard, F. Joucken, R. Sporken, F. Gargiulo, O. V. Yazyev, S. Rousset, Grain Boundaries in Graphene on $\mathrm{SiC}(000 \overline{1})$ Substrate, Nano Lett. 14 (11) (2014) 6382-6386 (Nov. 2014). doi:10.1021/n1502854w.

[8] G. Yang, L. Li, W. B. Lee, M. C. Ng, Structure of graphene and its disorders: A review, Science and Technology of Advanced Materials 19 (1) (2018) 613-648 (Dec. 2018). doi:10.1080/14686996. 2018. 1494493.

[9] E. Cockayne, G. M. Rutter, N. P. Guisinger, J. N. Crain, P. N. First, J. A Stroscio, Grain boundary loops in graphene, Phys. Rev. B 83 (19) (2011) 195425 (May 2011). doi : 10.1103/PhysRevB.83.195425.

[10] L. Linhart, J. Burgdörfer, F. Libisch, Accurate modeling of defects in graphene transport calculations, Phys. Rev. B 97 (3) (2018) 035430 (Jan. 2018). doi:10.1103/PhysRevB.97.035430.

[11] D. Kang, C. Zhang, H. Li, Spin Transport in Zigzag Graphene Nanoribbon with a Flower Defect, J Supercond Nov Magn 32 (12) (2019) $3927-$ 3931 (Dec. 2019). doi : 10.1007/s10948-019-05180-y.

[12] A. Cresti, J. Carrete, H. Okuno, T. Wang, G. K. H. Madsen, N. Mingo, P. Pochet, Growth, charge and thermal transport of flowered graphene, Carbon 161 (2020) 259-268 (May 2020). doi:10.1016/j.carbon. 2020.01 .040$.

[13] Y. Chen, M.-C. Li, Q.-M. Wang, G.-S. Wang, X. Wei, G.-F. Song, X.-M Kong, Y. Xu, Y. Liu, Structure and electronic properties of closed-ring defects in epitaxial graphene, Mater. Res. Express 7 (5) (2020) 055602 (May 2020). doi : 10.1088/2053-1591/ab8ee6.

[14] T. Björkman, S. Kurasch, O. Lehtinen, J. Kotakoski, O. V. Yazyev, A. Srivastava, V. Skakalova, J. H. Smet, U. Kaiser, A. V. Krasheninnikov, Defects in bilayer silica and graphene: Common trends in diverse hexagonal two-dimensional systems, Sci Rep 3 (1) (2013) 3482 (Dec. 2013). doi:10.1038/srep03482.

[15] M. Pivetta, M.-C. Blüm, F. Patthey, W.-D. Schneider, Two-Dimensional Tiling by Rubrene Molecules Self-Assembled in Supramolecular Pentagons, Hexagons, and Heptagons on a Au(111) Surface, Angew. Chem. Int. Ed. 47 (6) (2008) 1076-1079 (2008). doi:10.1002/anie. 200704479.

[16] B. An, S. Fukuyama, K. Yokogawa, Graphitization of $6 \mathrm{H}-\mathrm{SiC}(000 \backslash$ bar1 $)$ Surface by Scanning Tunneling Microscopy, Jpn. J. Appl. Phys. 41 (Part 1, No. 7B) (2002) 4890-4893 (Jul. 2002). doi:10.1143/JJAP. 41. 4890 .

[17] G. M. Rutter, J. N. Crain, N. P. Guisinger, T. Li, P. N. First, J. A. Stroscio, Scattering and Interference in Epitaxial Graphene, Science 317 (5835) (2007) 219-222 (Jul. 2007). doi:10.1126/science.1142882.

[18] N. P. Guisinger, G. M. Rutter, J. N. Crain, C. Heiliger, P. N. First, J. A. Stroscio, Atomic-scale investigation of graphene formation on $6 \mathrm{H}$ SiC(0001), J. Vac. Sci. Technol. A 26 (4) (2008) 932 (Jul. 2008). doi : 10.1116/1.2900661.

[19] L. Simon, C. Bena, F. Vonau, D. Aubel, H. Nasrallah, M. Habar, J. C. Peruchetti, Symmetry of standing waves generated by a point defect in epitaxial graphene, Eur. Phys. J. B 69 (3) (2009) 351-355 (Jun. 2009). doi:10.1140/epjb/e2009-00142-3.

[20] H. J. Park, V. Skákalová, J. Meyer, D. S. Lee, T. Iwasaki, C. Bumby, U. Kaiser, S. Roth, Growth and properties of chemically modified graphene, Phys. Stat. Sol. 247 (11-12) (2010) 2915-2919 (2010). doi : 10.1002/pssb. 201000818.

[21] H. I. Rasool, E. B. Song, M. Mecklenburg, B. C. Regan, K. L. Wang, B. H. Weiller, J. K. Gimzewski, Atomic-Scale Characterization of Graphene Grown on Copper (100) Single Crystals, J. Am. Chem. Soc. 133 (32) (2011) 12536-12543 (Aug. 2011). doi:10.1021/ja200245p

[22] H. Yan, C.-C. Liu, K.-K. Bai, X. Wang, M. Liu, W. Yan, L. Meng, Y. Zhang, Z. Liu, R.-f. Dou, J.-C. Nie, Y. Yao, L. He, Electronic structures of graphene layers on a metal foil: The effect of atomic-scale defects, App. Phys. Lett. 103 (14) (2013) 143120 (Oct. 2013). doi : 10.1063/1.4824206.

[23] Y. Cui, H. Zhang, W. Chen, Z. Yang, Q. Cai, Structural Evolution of Flower Defects and Effects on the Electronic Structures of Epitaxial Graphene, J. Phys. Chem. C 121 (28) (2017) 15282-15287 (Jul. 2017). doi:10.1021/acs.jpcc.7b04693.

[24] T. Hu, D. Ma, Q. Fang, P. Zhang, X. Liu, R. Wei, Y. Pan, K. Xu, F. Ma,
Bismuth mediated defect engineering of epitaxial graphene on $\mathrm{SiC}(0001)$, Carbon 146 (2019) 313-319 (May 2019). doi:10.1016/j.carbon. 2019.02 .014$.

[25] J. C. Meyer, C. Kisielowski, R. Erni, M. D. Rossell, M. F. Crommie, A. Zettl, Direct Imaging of Lattice Atoms and Topological Defects in Graphene Membranes, Nano Lett. 8 (11) (2008) 3582-3586 (Nov. 2008). doi: $10.1021 / \mathrm{nl} 801386 \mathrm{~m}$

[26] S. Kurasch, J. Kotakoski, O. Lehtinen, V. Skákalová, J. Smet, C. E. Krill, A. V. Krasheninnikov, U. Kaiser, Atom-by-Atom Observation of Grain Boundary Migration in Graphene, Nano Lett. 12 (6) (2012) 3168-3173 (Jun. 2012). doi : 10.1021/nl301141g.

[27] J. Kotakoski, J. C. Meyer, Mechanical properties of polycrystalline graphene based on a realistic atomistic model, Phys. Rev. B 85 (19) (2012) 195447 (May 2012). doi :10.1103/PhysRevB.85.195447.

[28] E. Cockayne, Graphing and grafting graphene: Classifying finite topological defects, Phys. Rev. B 85 (12) (2012) 125409 (Mar. 2012). doi : 10.1103/PhysRevB.85.125409.

[29] J. Zhuang, R. Zhao, J. Dong, T. Yan, F. Ding, Evolution of domains and grain boundaries in graphene: A kinetic Monte Carlo simulation, Phys. Chem. Chem. Phys. 18 (4) (2016) 2932-2939 (Jan. 2016). doi : 10.1039/C5CP07142A.

[30] H. Terrones, R. Lv, M. Terrones, M. S. Dresselhaus, The role of defects and doping in 2D graphene sheets and 1D nanoribbons, Rep. Prog. Phys. 75 (6) (2012) 062501 (May 2012). doi : 10.1088/0034-4885/75/6/ 062501.

[31] S. Karoui, H. Amara, C. Bichara, F. Ducastelle, Nickel-Assisted Healing of Defective Graphene, ACS Nano 4 (10) (2010) 6114-6120 (Oct. 2010). doi:10.1021/nn101822s.

[32] Y. Zhang, V. W. Brar, F. Wang, C. Girit, Y. Yayon, M. Panlasigui, A. Zettl, M. F. Crommie, Giant phonon-induced conductance in scanning tunnelling spectroscopy of gate-tunable graphene, Nature Phys 4 (8) (2008) 627-630 (Aug. 2008). doi:10.1038/nphys1022.

[33] P. Giannozzi, S. Baroni, N. Bonini, M. Calandra, R. Car, C. Cavazzoni, D. Ceresoli, G. L. Chiarotti, M. Cococcioni, I. Dabo, A. D. Corso, S. de Gironcoli, S. Fabris, G. Fratesi, R. Gebauer, U. Gerstmann, C. Gougoussis, A. Kokalj, M. Lazzeri, L. Martin-Samos, N. Marzari, F. Mauri, R. Mazzarello, S. Paolini, A. Pasquarello, L. Paulatto, C. Sbraccia, S. Scandolo, G. Sclauzero, A. P. Seitsonen, A. Smogunov, P. Umari, R. M. Wentzcovitch, QUANTUM ESPRESSO: A modular and opensource software project for quantum simulations of materials, J. Phys. Condens. Matter 21 (39) (2009) 395502 (Sep. 2009). doi:10.1088/ 0953-8984/21/39/395502.

[34] P. E. Blöchl, Projector augmented-wave method, Phys. Rev. B 50 (24) (1994) 17953-17979 (Dec. 1994). doi:10.1103/PhysRevB.50. 17953.

[35] J. P. Perdew, K. Burke, M. Ernzerhof, Generalized gradient approximation made simple, Phys. Rev. Lett. 77 (18) (1996) 3865-3868 (Oct. 1996). doi: 10.1103/PhysRevLett.77.3865.

[36] N. Mishra, J. Boeckl, N. Motta, F. Iacopi, Graphene growth on silicon carbide: A review, Phys. Stat. Sol. 213 (9) (2016) 2277-2289 (2016). doi:10.1002/pssa. 201600091.

[37] J. Hass, F. Varchon, J. E. Millán-Otoya, M. Sprinkle, N. Sharma, W. A. de Heer, C. Berger, P. N. First, L. Magaud, E. H. Conrad, Why Multilayer Graphene on $4 \mathrm{H}-\mathrm{SiC}(000-1)$ Behaves Like a Single Sheet of Graphene, Phys. Rev. Lett. 100 (12) (2008) 125504 (Mar. 2008). doi:10.1103/ PhysRevLett.100.125504.

[38] I. Forbeaux, J.-M. Themlin, J.-M. Debever, High-temperature graphitization of the 6H-SiC (0001) face, Surf. Sci. 442 (1) (1999) 9-18 (Nov. 1999). doi : 10.1016/S0039-6028(99)00891-2.

[39] A. Tiberj, J.-R. Huntzinger, J. Camassel, F. Hiebel, A. Mahmood, P. Mallet, C. Naud, J.-Y. Veuillen, Multiscale investigation of graphene layers on 6H-SiC(000-1), Nanoscale Res Lett 6 (1) (2011) 171 (Dec. 2011). doi : $10.1186 / 1556-276 \mathrm{X}-6-171$

[40] L. P. Biró, P. Lambin, Grain boundaries in graphene grown by chemical vapor deposition, New J. Phys. 15 (3) (2013) 035024 (Mar. 2013). doi : 10.1088/1367-2630/15/3/035024.

[41] L. Liu, Z. Chen, L. Wang, E. Polyakova (Stolyarova), T. Taniguchi, K. Watanabe, J. Hone, G. W. Flynn, L. E. Brus, Slow Gold Adatom Diffusion on Graphene: Effect of Silicon Dioxide and Hexagonal Boron Nitride Substrates, J. Phys. Chem. B 117 (16) (2013) 4305-4312 (Apr. 2013). doi : 10.1021/jp305521g. 
[42] X. Liu, C.-Z. Wang, M. Hupalo, H.-Q. Lin, K.-M. Ho, M. Tringides, Metals on Graphene: Interactions, Growth Morphology, and Thermal Stability, Crystals 3 (1) (2013) 79-111 (Jan. 2013). doi:10.3390/ cryst3010079.

[43] M.-Y. Li, Q. Zhang, P. Pandey, M. Sui, E.-S. Kim, J. Lee, From the Au nano-clusters to the nanoparticles on $4 \mathrm{H}-\mathrm{SiC}$ (0001), Sci. Rep. 5 (1) (Nov. 2015). doi:10.1038/srep13954.

[44] J. Zhang, J. Zhao, Mechanical properties of bilayer graphene with twist and grain boundaries, J. App. Phys. 113 (4) (2013) 043514 (Jan. 2013). doi:10.1063/1.4789594.

[45] B. Westenfelder, J. Biskupek, J. C. Meyer, S. Kurasch, X. Lin, F. Scholz, A. Gross, U. Kaiser, Bottom-up formation of robust gold carbide, Sci Rep 5 (1) (2015) 1-6 (Mar. 2015). doi : 10.1038/srep08891.

[46] S. Stolbov, M. Alcántara Ortigoza, Gold-doped graphene: A highly stable and active electrocatalysts for the oxygen reduction reaction, J. Chem. Phys. 142 (15) (2015) 154703 (Apr. 2015). doi:10.1063/1.4917468.

[47] F. Varchon, P. Mallet, L. Magaud, J.-Y. Veuillen, Rotational disorder in few-layer graphene films on $6 \mathrm{H}-\mathrm{Si} \mathrm{C}$ ( 000 - 1) : A scanning tunneling microscopy study, Phys. Rev. B 77 (16) (2008) 165415 (Apr. 2008). doi : 10.1103/PhysRevB.77.165415.

[48] W. Norimatsu, M. Kusunoki, Growth of graphene from $\mathrm{SiC}\{0001\}$ surfaces and its mechanisms, Semicond. Sci. Technol. 29 (6) (2014) 064009 (Jun. 2014). doi : 10.1088/0268-1242/29/6/064009.

[49] L. B. Biedermann, M. L. Bolen, M. A. Capano, D. Zemlyanov, R. G. Reifenberger, Insights into few-layer epitaxial graphene growth on $4 \mathrm{H}$ SiC(000-1) substrates from STM studies, Phys. Rev. B 79 (12) (2009) 125411 (Mar. 2009). doi:10.1103/PhysRevB.79.125411.

[50] F. Hiebel, P. Mallet, F. Varchon, L. Magaud, J.-Y. Veuillen, Graphenesubstrate interaction on $6 \mathrm{H}-\mathrm{SiC}$ ( 000-1): A scanning tunneling microscopy study, Phys. Rev. B 78 (15) (2008) 153412 (Oct. 2008) doi:10.1103/PhysRevB.78.153412.

[51] A. Pulido, M. Boronat, A. Corma, Theoretical investigation of gold clusters supported on graphene sheets, New J. Chem. 35 (10) (2011) 21532161 (2011). doi:10.1039/C1NJ20215D.

[52] T. Irawan, I. Barke, H. Hövel, Size-dependent morphology of gold clusters grown on nanostructured graphite, Appl. Phys. A 80 (5) (2005) 929935 (Feb. 2005). doi : 10.1007/s00339-004-3118-8.

[53] E. Annevelink, E. Ertekin, H. T. Johnson, Grain boundary structure and migration in graphene via the displacement shift complete lattice, Acta Materialia 166 (2019) 67-74 (Mar. 2019). doi:10.1016/j .actamat . 2018.12.030.

[54] K. T. Chan, J. B. Neaton, M. L. Cohen, First-principles study of metal adatom adsorption on graphene, Phys. Rev. B 77 (23) (2008) 235430 (Jun. 2008). doi:10.1103/PhysRevB.77.235430.

[55] K. Nakada, A. Ishii, Migration of adatom adsorption on graphene using DFT calculation, Solid State Commun. 151 (1) (2011) 13-16 (2011). doi:10.1016/j.ssc.2010.10.036.

[56] M. Manadé, F. Viñes, F. Illas, Transition metal adatoms on graphene: A systematic density functional study, Carbon 95 (2015) 525-534 (2015). doi:10.1016/j.carbon.2015.08.072.

[57] L. Li, S. Reich, J. Robertson, Defect energies of graphite: Densityfunctional calculations, Phys. Rev. B 72 (18) (2005) 184109 (Nov. 2005). doi:10.1103/PhysRevB.72.184109.

[58] J.-C. Charlier, H. Amara, P. Lambin, Catalytically Assisted Tip Growth Mechanism for Single-Wall Carbon Nanotubes, ACS Nano 1 (3) (2007) 202-207 (Oct. 2007). doi : 10.1021/nn700049q.

[59] W.-W. Wang, J.-S. Dang, J.-J. Zheng, X. Zhao, E. Ōsawa, S. Nagase, Metal-promoted restoration of defective graphene, J. Mater. Chem. 22 (32) (2012) 16370-16375 (Jul. 2012). doi : 10.1039/C2JM31703F.

[60] H. Amara, S. Latil, V. Meunier, P. Lambin, J.-C. Charlier, Scanning tunneling microscopy fingerprints of point defects in graphene: A theoretical prediction, Phys. Rev. B 76 (11) (2007) 115423 (Sep. 2007). doi:10.1103/PhysRevB.76.115423.

[61] A. Lherbier, S. M.-M. Dubois, X. Declerck, S. Roche, Y.-M. Niquet, J.C. Charlier, Two-Dimensional Graphene with Structural Defects: Elastic Mean Free Path, Minimum Conductivity, and Anderson Transition, Phys. Rev. Lett. 106 (4) (2011) 046803 (Jan. 2011). doi:10.1103/ PhysRevLett. 106.046803.

[62] O. V. Yazyev, S. G. Louie, Electronic transport in polycrystalline graphene, Nature Mater 9 (10) (2010) 806-809 (Oct. 2010). doi: $10.1038 /$ nmat 2830 .
[63] A. Luican-Mayer, J. E. Barrios-Vargas, J. T. Falkenberg, G. Autès, A. W. Cummings, D. Soriano, G. Li, M. Brandbyge, O. V. Yazyev, S. Roche, E. Y. Andrei, Localized electronic states at grain boundaries on the surface of graphene and graphite, 2D Mater. 3 (3) (2016) 031005 (Aug. 2016). doi : 10.1088/2053-1583/3/3/031005.

[64] J. Červenka, K. van de Ruit, C. F. J. Flipse, Local enhancement of inelastic tunnelling in epitaxial graphene on SiC(0001), Phys. Stat. Sol. B 247 (11-12) (2010) 2992-2996 (Dec. 2010). doi:10.1002/pssb. 201000167.

[65] C. Tao, L. Jiao, O. V. Yazyev, Y.-C. Chen, J. Feng, X. Zhang, R. B. Capaz, J. M. Tour, A. Zettl, S. G. Louie, H. Dai, M. F. Crommie, Spatially resolving edge states of chiral graphene nanoribbons, Nature Phys 7 (8) (2011) 616-620 (Aug. 2011). doi:10.1038/nphys1991.

[66] M. Ridene, J. C. Girard, L. Travers, C. David, A. Ouerghi, STM/STS investigation of edge structure in epitaxial graphene, Surf. Sci. 606 (15) (2012) 1289-1292 (Aug. 2012). doi : 10.1016/j . susc. 2012.04.006.

[67] M. Mirzakhani, F. M. Peeters, M. Zarenia, Circular quantum dots in twisted bilayer graphene, Phys. Rev. B 101 (7) (2020) 075413 (Feb. 2020). doi:10.1103/PhysRevB.101.075413. 